\title{
FraTURAS DO CÔNDILO MANDIBULAR: ANÁLISE CLÍNICA RETROSPECTIVA DAS INDICAÇŐES E DO TRATAMENTO
}

Eduardo Fausto de Andrade Filho*, Dulce Maria Fonseca Soares Martins, Miguel Sabino Neto, Carlos de Souza Toledo Júnior, Max Domingues Pereira, lydia Massako Ferreira

Trabalho realizado na Disciplina de Cirurgia Plástica do Departamento de Cirurgia da Universidade Federal de São Paulo - UNIFESP-EPM, São Paulo, SP

RESUMO - Objetivo. Analisamos 40 pacientes com fraturas no processo condilar da mandíbula, tratados pelo método fechado (bloqueio maxilomandibular ou conservador) ou aberto (redução direta no foco de fratura com ou sem osteossíntese), no período de janeiro de 1993 a janeiro de 1999 no Setor de Trauma Maxilofacial da Disciplina de Cirurgia Plástica da UNIFESP-EPM.

Método. Avaliamos clinicamente estes pacientes através da palpação da articulação têmporomandibular, observação da simetria mandibular, oclusão dentária, desvio à abertura de boca e abertura máxima interincisal; além de questionário sobre queixas na ATM, disfunção mastigatória e satisfação do tratamento realizado.

Resultados. Foi encontrado um índice de resultados satisfatórios de no mínimo $70 \%$ nos pacientes submetidos ao bloqueio. No método conservador esse índice foi de no mínimo $42,9 \%$, e nos submetidos à redução com fio de aço de no mínimo $28,6 \%$, sem diferença significante entre os diversos exames clínicos. 0 índice de dor na ATM entre os pacientes tratados pelo método fechado foi de $3,7 \%$ e pelo aberto de $15,4 \%$. A disfunção mastigatória foi de $22,2 \%$ no método fechado e $15,4 \%$ no aberto; sendo que foram encontrados somente $7,1 \%$ de pacientes insatisfeitos com 0 tratamento fechado. Não houve diferença significante entre os resultados dos métodos.

Conclusão. Os métodos fechado (BMM e conservador) e aberto, com redução e osteossíntese com fio de aço, trouxeram resultados clínicos satisfatórios em avaliação superior a seis meses. Concluiuse também que não há diferença entre os resultados de queixas após o tratamento aberto ou fechado, na opinião dos pacientes.

UNITERMOS: Trauma. Fraturas côndilo mandibular. Tratamento.

\section{INTRODUÇÃO}

Traumas na região mandibular que resultem em fraturas do processo condilar da mandíbula podem levar a alterações não somente no desenvolvimento facial, como na oclusão dentária e na movimentação da articulação temporomandibular(ATM).

Desse modo, o diagnóstico das fraturas ea indicação do tratamento devem ser precisos para se evitar as disfunções dessa articulação ou ainda seqüelas mais graves que alterem 0 crescimento da face e da mandíbula ou impossibilitem a abertura satisfatória da boca, como as anquiloses temporomandibulares.

Aindicação e o tratamento das fraturas do côndilo mandibular ainda são temas controversos nos dias de hoje, em que diversos autores defendem o método fechado por acreditarem numa remodelação do côndilo mandibular, principalmente em crianças me-

* Correspondência:

Rua Cônego Eugênio Leite, 693-apto 101

Pinheiros - São Paulo-SP-CEP: 05414011

Tel: 30833583-fax: 50522570-cel: 99890610

E-mail:eduandradefilho@zipmail.com.br nores de 12 anos ${ }^{1,2,3,4}$. Por outro lado, por observarem alterações clínicas tardias nos pacientes tratados com método fechado, há os que indicam o aberto em casos determinados, com exploração direta do local da fratura, redução e osteossíntese $e^{5,6,7,8}$

Este estudo tem como objetivo analisar as indicações de tratamento e avaliar clinicamente 40 pacientes com fraturas no processo condilar da mandíbula submetidos ao tratamento fechado ou aberto, num período de observação de, no mínimo, seis meses.

\section{Métodos}

Foi realizado um estudo retrospectivo, de janeiro de 1993 a janeiro de 1999, em que foram avaliados 40 pacientes vítimas de trauma mandibular com fraturas de côndilo, atendidos no setor de trauma Maxilofacial da Disciplina de Cirurgia Plástica do Departamento de Cirurgia da Universidade Federal de São Paulo EPM. A avaliação pós-tratamento variou de no mínimo seis meses até 72 meses.

Dos 40 pacientes analisados, 29 eram do sexo masculino $(72,50 \%)$ e II do feminino
(27,50\%), perfazendo um índice masculino/feminino de 2,63. A idade variou de 4 a 92 anos, com mediana de 22 anos. A faixa etária mais acometida foi a dos 10 - 19 anos (32,50\%).

As etiologias das fraturas de côndilo foram classificadas em acidentes de tráfego, (incluindo o automobilístico, de bicicleta e atropelamentos); quedas de alturas e em nível do chão, violência física e acidentes de trabalho, baseadas na Classificação Internacional de Doenças.

Os acidentes de tráfego foram a causa mais comum das fraturas perfazendo, 20 pacientes (50\%), seguidos das quedas com $13(32,50 \%)$ e agressões com cinco pacientes ( $12,50 \%)$. Dois pacientes sofreram trauma por acidentes de trabalho (5\%).

Foram submetidos a tratamento através do método fechado (bloqueio maxilomandibular ou conservador) 27 pacientes $(65,50 \%)$ e 13 pacientes $(32,50 \%)$ pelo aberto (exploração direta do foco com ou sem fixação).

Bloqueio maxilomandibular (BMM), isoladamente, foi o método de tratamento utilizado em 20 pacientes (50\%), seguido da redução e osteossíntese com fio de aço associado ao BMM em sete pacientes (I7,50\%) e do con- 


\begin{tabular}{llccc}
\hline \multicolumn{4}{l}{ Tabela I - Distribuição segundo tratamento realizado nos pacientes com fraturas de côndilo mandibular } \\
\hline Tratamento & Número & Total & $\%$ \\
Fechado & Bmm* & 20 & & \\
& Conservador & 7 & & \\
Aberto & Ost. FiodeAço** & 7 & 27 & 67,50 \\
& Ost. MiniplacadeTitânio & 3 & & \\
& Reposicionamentónsseo & 2 & & \\
& ExploraçâaArticular & 1 & 13 & 32,50 \\
& & 40 & 40 & 100,00 \\
\hline
\end{tabular}

- BMM-Bloqueio Maxilomandibular; **OST-Osteossíntese

\begin{tabular}{|c|c|c|c|c|c|}
\hline Paciente $\mathbf{N}^{\circ}$ & A & B & C & D & $\mathbf{E}$ \\
\hline 1 & t & + & - & + & - \\
\hline 3 & + & + & + & t & + \\
\hline 5 & + & - & + & + & + \\
\hline 8 & t & + & t & + & + \\
\hline 12 & + & + & t & + & + \\
\hline 13 & + & t & t & t & + \\
\hline 15 & + & + & + & + & + \\
\hline 16 & - & t & t & t & t \\
\hline 17 & + & + & - & + & t \\
\hline 18 & + & + & t & + & + \\
\hline 23 & + & t & t & t & + \\
\hline 24 & + & + & t & + & + \\
\hline 25 & - & + & t & t & t \\
\hline 26 & + & + & t & + & + \\
\hline 30 & - & t & t & - & - \\
\hline 33 & - & + & t & - & + \\
\hline 35 & t & t & t & - & - \\
\hline 37 & t & - & t & - & - \\
\hline 38 & + & + & t & - & + \\
\hline 40 & + & - & t & - & - \\
\hline TOTAL ( +) & $(+) 1680,0$ & 1785,0 & 1890,0 & 1470,0 & 1575,0 \\
\hline
\end{tabular}

Total $(+)$ : somatória da presença $(+) ; \%(+)$ : percentual da somatória da presença $(+)$

Teste "G" de Cochran $\quad G_{\text {CALCULAdO }}=3,57 G_{\text {CRITICO }}=9,49$

servador com dieta líquida e pastosa na mesma proporção. Osteossíntese com miniplaca de titânio foi realizada em três pacientes, e ainda outros dois obtiveram reposicionamento anatômico ósseo sem osteossíntese e uma exploração da articulação temporomandibular com liberação de aderência óssea (Tabela I).

\section{Métodos}

Independentemente do tipo de tratamento realizado, os 40 pacientes foram submeti- dos a avaliação clínica e das Fichas de Trauma específicas do Setor de Trauma Maxilofacial.

Foi criada ficha complementar com dados de medidas dos movimentos da mandíbula dos pacientes e de interrogatório sobre queixas de dor, disfunção mastigatória e satisfação póstratamento. Os sinais e sintomas existentes foram considerados somente após o trauma e seu tratamento.

Osmétodos de exame clínico einterrogatórioforambaseadosnoestudo de Helkimo $(1974)^{9}$ sobre a função e a disfunção do sistema mastigatório, semescala de pontuação esimplificado, considerando resultados satisfatórios e insatisfatórios, conforme critériosadotados.

\section{Exame clínico pós-tratamento}

O exame clínico incluiu:

A) Palpação da articulação temporomandibular com boca aberta e fechada com resultado satisfatório quando não existia dor ou estalidos e insatisfatório na presença destes sinais ou sintomas.

B) Observação da simetria mandibular com resultado satisfatório quando não existia assimetria e insatisfatório na presença de assimetria visível.

C) Observação da oclusão dentária com resultado satisfatório na oclusão semelhante a anterior ao trauma e insatisfatório na presença de desvio, mordida cruzada ou aberta.

D) Abertura de boca (interincisal máxima) com resultado satisfatório quando maior ou igual a $40 \mathrm{~mm}$ e insatisfatório quando inferior a essa medida.

E) Desvio da linha média à abertura de boca com resultado satisfatório quando ausente ou menor que $2 \mathrm{~mm}$ e insatisfatório quando presente ou maior que $2 \mathrm{~mm}$.

\section{Interrogatório aos pacientes}

Por meio da ficha complementar, um interrogatório foi realizado com os seguintes itens:

A) Dor em articulação temporomandibular ao abrir ou fechar a boca

B) Disfunção mastigatória ao alimentar.

C) Satisfação do tratamento realizado.

Consideramos como resultado satisfatório quando o mesmo não referia sintomatologia de dor, disfunção mastigatória, e apresentava satisfação com o tratamento realizado e insatisfatório quando apresentava queixa de dor, disfunção mastigatória ou encontrava-se insatisfeito com o tratamento realizado.

\section{Resultados}

Os resultados do exame clínico foram submetidos ao teste $G$ de Cochran ${ }^{10} \mathrm{e}$ os resultados do interrogatório ao teste exato de Fisher $^{10}$.

Em relação ao resultado do tratamento fechado com BMM (Tabela 2), o teste $G$ de Cochran não mostrou diferença entre as 
Tabela 3 - Pacientes com fraturas do côndilo mandibular, submetidos a tratamento conservador, segundo a presença de resultados satisfatórios (+) ou insatisfatórios (-), na palpação da ATM (A), simetria mandibular (B), oclusão dentária (C), abertura de boca (D) e desvio da linha média (E)

\begin{tabular}{|c|c|c|c|c|c|}
\hline Paciente $\mathrm{N}^{\circ}$ & A & B & C & D & $\mathbf{E}$ \\
\hline 6 & - & $t$ & - & - & - \\
\hline 10 & + & + & + & + & + \\
\hline 14 & - & + & + & + & + \\
\hline 19 & + & + & + & + & - \\
\hline 29 & - & $t$ & - & + & + \\
\hline 34 & - & $t$ & $t$ & - & + \\
\hline 36 & + & + & + & - & + \\
\hline TOTAL $(+) \% \quad(+)$ & 342,9 & 7100,0 & 571,4 & 457,1 & 571,4 \\
\hline
\end{tabular}

Total $(+)$ : somatória da presença $(+) ; \%(+)$ : percentual da somatória da presença $(+)$

Teste " $G$ " de Cochran $\quad G_{\text {CALCULADO }}=6,29 G_{\text {CRTICO }}=9,49$

Tabela 4 - Pacientes com fraturas do côndilo mandibular, submetidos a tratamento aberto com fio de aço, segundo a presença de resultados satisfatórios (+) ou insatisfatórios (-), na palpação da ATM (A), simetria mandibular (B), oclusão dentária (C), abertura de boca (D) e desvio da linha média $(E)$

\begin{tabular}{|c|c|c|c|c|c|}
\hline Paciente $\mathbf{N}^{\circ}$ & A & B & C & D & $\mathbf{E}$ \\
\hline 4 & - & - & + & - & + \\
\hline 9 & - & + & + & - & + \\
\hline II & - & + & + & - & + \\
\hline 20 & + & + & + & + & - \\
\hline 21 & + & + & + & + & + \\
\hline 28 & - & + & + & + & + \\
\hline 39 & - & - & + & + & + \\
\hline TOTAL $(+) \% \quad(+)$ & 228,6 & 571,4 & 7100,0 & 757,1 & 685,7 \\
\hline
\end{tabular}

Tabela 5 - Pacientes com fraturas do côndilo mandibular com tratamento aberto ou fechado, segundo relato das queixas em relação a dor em ATM

\begin{tabular}{|c|c|c|c|c|}
\hline Tratamento & $\begin{array}{c}\text { Dor } \\
\text { Presença }\end{array}$ & Ausência & Total & $\%$ Presença \\
\hline Fechado & I & 26 & 27 & 3,7 \\
\hline Aberto & 2 & II & 13 & 15,4 \\
\hline Total & 3 & 37 & 40 & 7,5 \\
\hline
\end{tabular}

Teste Exato de Fisher $P=0,2421$

Tabela 6 - Pacientes com fraturas do côndilo mandibular com tratamento aberto ou fechado, segundo relato das queixas em relação a disfunção mastigatória

\begin{tabular}{|c|c|c|c|c|}
\hline \multirow[t]{2}{*}{ Tratamento } & \multicolumn{2}{|c|}{ Disfunção } & \multirow[t]{2}{*}{ Total } & \multirow[t]{2}{*}{$\%$ Presença } \\
\hline & Presença & Ausência & & \\
\hline Fechado & 6 & 21 & 27 & 22,22 \\
\hline Aberto & 2 & II & 13 & 15,40 \\
\hline Total & 8 & 32 & 40 & 20,00 \\
\hline
\end{tabular}

Teste Exato de Fisher $P=0,4792$ percentagens de resultados satisfatórios observados nos vários exames clínicos. O exame com menor percentagem de resultados satisfatórios foi o de abertura de boca (interincisal máxima) com $70 \%$.

Em relação ao resultado do tratamento conservador não houve diferença estatísticamente significante entre os diversos exames realizados. Os dados sugerem, porém, que a presença de dor e estalidos em ATM foi maior que os outros exames clínicos realizados. A Tabela 3 demonstra esses resultados.

O tratamento aberto com fio de aço, demonstrado na Tabela 4, também não apresentou diferença estatística entre os exames realizados. Em função do tamanho da amostra não foi possível rejeitar a igualdade entre a presença de resultados satisfatórios, entretanto, os dados sugerem que a presença de dor e estalidos em ATM foi maior que as demais características.

\section{Resultados da análise em relação ao interrogatório}

Por se tratar de interrogatório subjetivo sobre queixas e satisfação dos pacientes, foram comparados os grupos fechado e aberto em relação à presença de dor na abertura de boca, à disfunção mastigatória eà satisfação do tratamento realizado. As Tabelas 5 a 7 apontam esses dados.

Em relação à essas queixas, o Teste Exato de Fischer não mostrou diferença significante entre os dois tipos de tratamento.

\section{Discussão}

Apesar da mandíbula ser um dos locais mais atingidos por traumas na região facial, as fraturas do processo condilar muitas vezes não são diagnosticadas, em especial quando são atingidos o colo e a cabeça condilar.

Ainda não existe consenso na indicação do tratamento utilizado e na evolução clínica desses pacientes, não somente pela dificuldade de homogenidade das amostras, como de controle pós-tratamento, além dos diversos fatores que influenciam no seu diagnóstico e principalmente na forma de tratamento, o que nos motivou a analisar as indicações e avaliar as evoluções clínicas dos pacientes tratados em cada método.

Diversos são os fatores que influenciam na indicação e, consequentemente, no prognós- 
Tabela 7 - Pacientes com fraturas do côndilo mandibular com tratamento aberto ou fechado, segundo relato das queixas em relação à satisfação do tratamento

\begin{tabular}{lcccc}
\hline Tratamento & \multicolumn{2}{c}{ Satisfação } & Total & \% Presença \\
& Presença & Ausência & & \\
\cline { 2 - 3 } Fechado & 25 & 2 & 27 & 92,59 \\
Aberto & 13 & 0 & 13 & 100,0 \\
Total & 38 & 2 & 40 & 95,0 \\
\hline
\end{tabular}

Teste Exato de Fisher $\mathrm{P}=0,4500$

tico do tratamento das fraturas condilares e devem ser levados em consideração antes da decisão final sobre o tipo de tratamento; ressaltando-se que não devem ser avaliados isoladamente, mas sim em conjunto.

Podem ser citados o grau de deslocamento do fragmento, idade do paciente, localização da fratura, direção do deslocamento, estado físico do paciente, fraturas associadas no terço médio da face, presença e condições da dentição, possibilidade de realizar oclusão adequada, presença de corpos estranhos na articulação temporomandibular e trauma com lesão exposta, como descritos por Zide e Kent".

Foram considerados indicados ao tratamento fechado com BMM pacientes que apresentavam má oclusão dentária, desvio do fragmento menor do que aproximadamente $45^{\circ}$, quando o processo condilar permanecia dentro da cavidade glenóide, quando apresentava elementos dentários suficientes para o BMM ou quando da ausência dos dentes havia possibilidade de utilização de próteses.

A dieta líquida e pastosa (tratamento conservador) foi orientada como tratamento em pacientes com oclusão inalterada após o trauma, sem desvio do fragmento fraturado e sem limitação à abertura de boca, ou ainda em pacientes sem condições clínicas de tratamento cirúrgico.

Ogrupo de pacientes submetidos ao tratamento fechado representou a maioria (67,50\%) do total de pacientes tratados, como já demonstraram diversos autores $3,12,13,14,15,16$.

O tratamento aberto foi realizado em pacientes que apresentavam desvios do côndilo maiores que aproximadamente $45^{\circ}$, côndilo fora da cavidade articular, fraturas cominutivas, fragmentos de corpos estranhos intra-articulares e bloqueio à movimentação da ATM. Neste grupo foram tratados $32,5 \%$ dos pacientes.

Pudemos observar que o grupo tratado com BMM apresentou percentual homogêneo de resultados satisfatórios sem apresentar diferença estatística entre as diferentes avaliações clínicas realizadas, porém sugerindo que o exame clínico de abertura de boca tenha obtido o menor número de bons resultados, com $30 \%$ dos pacientes com abertura menor que $40 \mathrm{~mm}$. de idades variadas com fraturas do côndilo mandibulares tratados pelo método conservador com BMM ou fisioterapia, e encontraram, após um ano do final do tratamento, 45 pacientes (13\%) com queixas fisiológicas menores de redução na abertura máxima interincisal, desvio a abertura de boca ou disfunções da ATM. Referem que este tratamento é seguro, não traumático e que as complicações mais freqüentes ocorrem em pacientes com fraturas bilaterais e com deslocamento da fossa articular.

É interessante notar que desse grupo de seis pacientes com abertura menor que 40 $\mathrm{mm}$, cinco estão na faixa de 12 a 18 anos de idade, o que nos reporta aos trabalhos de Lindhal ${ }^{17}$, que descrevem que os adolescentes podem apresentar resultados insatisfatórios por ocuparem posição intermediária no mecanismo de remodelação do processo condilar após o trauma.

Esses pacientes não foram agrupados com outros de mesma faixa etária para que pudéssemos comparar os resultados dos tratamentos, pela impossibilidade de padronização dos diversos fatores que influenciam na indicação do tratamento, como a diferença dos locais de fratura, grau de deslocamento do côndilo. Eles, porém, tiveram as mesmas indicações para inclusão neste tipo de tratamento e observamos que evoluíram de modo diverso.

Um estudo com as mesmas características de idade, local de fratura e deslocamento, com diferentes tipos de tratamen-
Marker et al. ${ }^{4}$ analisaram 348 pacientes tos, é necessário para que essas variáveis não influenciem no resultado.

É importante ressaltar que não se configurou como objetivo desse estudo comparar os diferentes tipos de tratamento, e sim observarmos os resultados clínicos obtidos conforme nossas indicações baseadas na literatura e na nossa experiência.

Era por nós esperado que os resultados satisfatórios fossem obtidos, em sua maioria, através do método fechado com BMM, por se tratar de um método de tratamento consagrado e de pacientes com fraturas menos graves, com menor desvio do fragmento.

A análise estatística sugere que, nesse tipo de tratamento (BMM), os pacientes podem apresentar menor quantidade de resultados satisfatórios no exame clínico de abertura de boca do que em relação aos outros exames clínicos realizados. Isso reforça a idéia da necessidade de exames mais precisos, como ressonância magnética ou artroscopia para melhor diagnóstico de lesão intracapsular e posicionamento do disco articular, já que a lesão das estruturas intracapsulares pode ocorrer, mesmo em pequenas fraturas do côndilo, como sugerido por Goss e Bosanquet ${ }^{18}$; Jones e Sickels ${ }^{19}$ e Sullivan et al. ${ }^{20}$.

Outro fator para darmos atençãoéa posição que o disco articular pode adquirir após o trauma, trazendo sintomatologia nas fraturas com e sem deslocamento. Novamente, exames como a ressonância magnética podem ser necessários, auxiliando no diagnóstico do trauma e na evolução do tratamento para melhor compreensão da evolução de toda dinâmica da ATM, e não apenas na preocupação da consolidação óssea do fragmento do côndilo.

Pelo método conservador, a análise não mostrou diferença entre os resultados, porém sugere que os resultados clínicos de palpação da ATM tenham trazido menor quantidade de resultados satisfatórios. Apesar desse grupo ser de pacientes com os menores desvios do côndilo e sem alterações de oclusão (com exceção do paciente número seis), tivemos aproximadamente $50 \%$ dos casos apresentando dor ou estalidos em ATM.

Devemos lembrar que nesse grupo de sete pacientes, seis tinham idade entre $42 \mathrm{e}$ 96 anos, o que poderia justificar as alterações de ATM pela própria idade, tornando difícil de determinarmos a real etiologia desses sintomas. 
Uma avaliação clínica apurada deve ser feita com atenção especial à ATM, seus sintomas e sinais, assim como a história anterior ao trauma para que se possa determinar se suas alterações são decorrentes do próprio trauma ou não.

Apesar de o grupo de pacientes tratados com redução e osteossíntese com fio de aço ser de pacientes com fraturas mais graves, com maiores deslocamentos, a quantidade de bons resultados nos pareceu adequada e em concordância com os trabalhos de Hidding et al. ${ }^{21}$; Konstantinovic e Dimitrijevic ${ }^{15} \mathrm{e}$ Worsaae e Thorn ${ }^{22}$.

É importante ressaltar que não foi encontrado nenhum paciente tratado pelo método aberto com lesão nervosa definitiva, somente temporária no paciente de número 23 com recuperação total em quatro meses.

Não obstante, Ellis III ${ }^{23}$ referiu que a lesão nervosa pode ser encontrada em até $15 \%$ dos casos tratados cirurgicamente, com recuperação em torno de seis meses, porém lesões definitivas podem ocorrer com prejuízo funcional ao paciente.

No grupo de pacientes tratados pelo método aberto, apresentando idades variadas, pudemos notar que os pacientes com idade inferior a dez anos obtiveram menor quantidade de resultados satisfatórios. Desse modo, podemos nos reportar aos autores que defendem somente $o$ tratamento conservador em crianças abaixo de 12 anos, como Lindhal ${ }^{17}$ ou Dalström et al. ${ }^{2}$, que acreditam que o trauma cirúrgico provocado nas estruturas da ATMe do próprio côndilo pode alterar o crescimento mandibular, a vascularização e a anatomia da região.

Nossa preocupação no tratamento cirúrgico nesses casos foi a de restabelecer a anatomia da estrutura óssea e da relação do côndilo na articulação, com o intuito de evitar consolidações ósseas em posição não anatômica que pudessem provocar seqüelas, como as anquiloses temporomandibulares.

Outro fator relevanteé que nesses pacientes tratados pelo método aberto, a desinserção da musculatura do pterigóide lateral ocorreu onde o fragmento ósseo reposicionado funcionará como um enxerto, podendo sofrer absorção e conseqüentes alterações clínicas na função articular.

Pereira et al. ${ }^{8}$ estudaram 17 pacientes com fratura e deslocamento do côndilo, tratados pelo método aberto e fixação com fio de aço, com e sem desinserção da musculatura do pterigóide lateral. Observaram, à tomografia computadorizada, sinais de absorção óssea com perda da altura vertical do ramo mandibular, principalmente nos pacientes que sofreram desinserção muscular.

$\mathrm{Na}$ comparação entre os métodos fechado e aberto, em relação às queixas do pacientes, não encontramos diferenças estatísticas, reafirmando que nossas indicações trouxeram bons resultados em ambos os métodos na maioria dos casos.

Observamos durante a realização deste estudo a grande importância que existe em protocolarmos os pacientes com fraturas de côndilo, seus resultados e as indicações de tratamento mais adequado para cada caso.

Baker, $\mathrm{MCmahon}$, Moos ${ }^{24}$ realizaram questionário com 58 médicos da Associação Internacional de Cirurgia Oral e Maxilofacial, avaliando estratégias de tratamento para pacientes com fraturas de côndilo. Observou-se consenso no tratamento das fraturas com pouco desvio através do BMM, porém opiniões diversas em relação ao tratamento, se aberto ou fechado, para as fraturas com deslocamentos do processo condilar.

Existem na literatura poucos estudos comparativos do tipo de tratamento, devido à grande quantidade de variáveis existentes nos pacientes com fraturas de côndilo, além de condutas de tratamento diversas e preferências pessoais como os trabalhos de Hiding $\mathrm{G}^{21} \mathrm{e}$ Worsaae e Thorn ${ }^{22}$.

Esse estudo não teve como objetivo de comparar os métodos utilizados, nem de determinar qual seria o método de tratamento mais adequado, pois a grande quantidade de variáveis que acompanhavam os pacientes não permite essas conclusões.

Estudos com grupos de mesma faixa etária, tipo de fratura, deslocamento semelhante e tempo de evolução deverão ser realizados num futuro próximo, com técnicas mais aperfeiçoadas, e com melhor diagnóstico da lesão intracapsular, para que possamos determinar uma conduta terapêutica cada vez mais adequada, objetivando melhores resultados funcionais a esses pacientes.

\section{Conclusão}

Através de nosso estudo pudemos concluir que o método de tratamento fechado, através do bloqueio maxilomandibular, no grupo de pacientes com fraturas do processo condilar selecionados para receber esse tratamento num período de observação de no mínimo seis meses, apresentou resultados satisfatórios na maioria das avaliações clínicas realizadas. O método de tratamento conservador mostrou-se com maior tendência de resultados insatisfatórios no exame clínico da ATM, assim como os pacientes tratados através da redução e osteossíntese com fio de aço. Não existe diferença estatisticamente significante no interrogatório aos pacientes nas queixas de dor, disfunção mastigatória e satisfação após o tratamento aberto ou fechado.

\section{SUMMARY}

\section{EVALUATION OF CONDYLAR FRACTURES TREATMENT}

BACKGROUND. We analysed 40 patients with mandibular condylar process fractures, treated through the closed (intermaxilary fixation or conservatively) or open methods (wire internal fixation, titanium miniplates, bone reduction or articular exploration), from January 1993 to January 1999.

Methods. We evaluated these patients clinically with pre-determined criteria through the temporomandibular articulation touching (ATM), by observing the mandibular symmetry, dental oclusion, deviation to the mouth opening and maximum interincisal opening, besides the questionnaire about the complaints at ATM, disfunction of the mastication system and satisfaction of the treatment. We found an index of good clinical results of, at least, $70 \%$ (in the maximum interincisal opening) in the patients submitted to IMF. In the conservative method, we found an index of, at least, $42.9 \%$ and in the patients submitted to wire open reduction, at least, $28.6 \%$ (in the ATM touching exam) without significant difference in the clinical exams; the pain index at ATM in the closed method was $3.7 \%$ and in the open one was $15.4 \%$; mastication disfunction was $22.2 \%$ in the closed method and $15.4 \%$ in the open one; we only found $7.1 \%$ dissatisfied patients in the closed method. There was no significant difference in the method results.

ConcLusIONs. The closed method - with IMF 
and conservative - and the open reduction with ORIF - brought good clinical results in an evaluation up to 6 months. We could also conclude that there is no difference in the complaint results after the open or closed treatment according to the patients' opinion. [Rev Assoc Med Bras 2003; 49(I): 54-9]

KEYWORDS: Trauma. Condylar fractures. Treatment.

\section{RefERÊNCIAS}

I. LindahI L. Condylar fractures of the mandible. I. Classification and relation to age, occlusion, and concomitant injuries of teeth and teethsupporting structures, and fractures of of the mandibular body. Int J Oral Surg 1977; 6:2-2I.

2. Dahlström L, Kahnberg KE, LindahI L. 15 years follow-up on condylar fractures. Int J Oral Maxillofac. Surg 1989; 18: 18-23.

3. Feifel H, Albert-Deumlich J, Riediger D. Long Ter follow-up of subcondylar fractures in children by electronic computer-assisted recording of condylar movements. Int J Oral Maxillofac Surg 1992; 21:70-6.

4. Marker $P$, Nielsen A, Lehmann Bastian $H$. Fractures of the mandibular condyle. Part 2: results of 348 patients. Br. J. Maxillofac. Surg 2000; 38:422-6.

5. Koberg WR, Momma WG. Treatment of fractures of the articular process by functional stable osteosynthesis using miniaturized dynamic compression plates. Int J Oral Surg 1978; 7: 256-62.

6. Chuong R, Piper MA. Open reduction of condilar fractures of the mandible in conjunction with repari of discal injury: a preliminary report. J Oral Maxillofac Surg 1988; 46:257-63.

7. Sargent L, Green JFJr. Plate and screw fixation of selected condylar fractures of the mandible. Ann Plast Surg 1992; 28:235-4 I.

8. Pereira MD, Marques A, Ishizuka M, Keira SM, Brenda E, Wolesker AB. Surgical treatment of the fractured and deslocated condylar process of the mandible. J Craniomaxillofac Surg 1995; 23:369-76.

9. Helkimo M. Studies on function and disfunction of the mastigatory system. Swed Dental I 974; 67:101-19.

10. Bunchaft G, Kellner SRO. Estatística sem mistério. Petrópolis: Editora Vozes; 1999.v.4, 9l0p.

II. Zide MF, Kent JN. Indications for open reduction of mandibular condyle fractures. J Oral Maxillofac Surg 1 983; 41:89-98.

12. Zou ZJ, Wu WT, Sun GX, Zhu XP, Zhang KH, Wu QG, et al. Remodeling of the temporomandibular joint after conservative treatment of condylar fractures. Dentomaxillofac Radiol 1987; 16:91-8.

13. Ozeki HTS. Technical note: guide groove for pinning subcondylar fractures. Br JOral Maxillofac Surg 199 |; 29:2 I I-2.

14. Yasuoka T, Oka N. Histomorphometric study of trabecular bone remodeling during condylar process fracture healing in the growing period : experimental study. J Oral Maxillofac Surg 1991; 49:98I-8.

15. Konstantinovic VS, Dimitrijevic B. Surgical versus conservative treatment of unilateral condylar process fractures; clinical and radiographic evaluation of 80 patients. J Oral Maxillofac Surg 1992; 50:340-52.

16. Silvennoinen $U$, lizuka $T$, Lindqvist $C$,
Oikarinen K. Different patterns of condylar fractures: na analysis of 382 patients in a 3 year period. J Oral Maxillofac Surg 1 992; 50: I 32-7.

17. Lindahl L, Hollender L. Condylar fractures of the mandible. II. A radiographic study of remodeling processes in the temporomandibular joint. Int J Oral Surg 1 977; 6: 153-65.

18. Goss NA, Bosanquet AG. The arthroscopic appearance of acute temporomandibular joint trauma.J Oral Maxillofac Surg 1990; 48:780-3.

19. Jones JK, Van Sickels JE. A preliminary report of arthroscopic findings following acute condylar trauma. J. Oral Maxillofac Surg 1991; 48:55-60.

20. Sullivan SM, Banghart PR, Anderson Q. Magnetic ressonance imaging assessment of acute soft tissue injuries to the temporomandibular joint. J Oral Maxillofac Surg 1995; 53:763-6.

21 . Hidding J, Wolf R, Pingel D. Surgical versus non-surgical treatment of fractures of the articular process of the mandible. J Craniomaxillofac Surg 1992; 20:345-7.

22. Worsaae N, Thorn JJ. Surgical versus nonsurgical treatment of unilatera dislocated low subcondylar fractures: a clinical study of 52 cases. J Oral Maxillofac Surg 1994; 52:253-60.

23. Ellis III E. Complications of mandibular condyle fractures. Int J Oral Maxillofac Surg 1998; 27:255-7.

24. Baker AW, McMahon J, Moss F. Current consensus on the management of fractures of the mandibular condyle. Int J Oral Maxillofac Surg 1988; 27:258-66.

Artigo recebido: $27 / 1$ I/200 I Aceito para publicação: I6/04/2002 